\title{
BMJ Open COBI (COntinuous hyperosmolar therapy for traumatic Brain-Injured patients) trial protocol: a multicentre randomised open-label trial with blinded adjudication of primary outcome
}

\author{
Antoine Roquilly, ${ }^{1}$ Sigismond Lasocki, ${ }^{2}$ Jean Denis Moyer, ${ }^{3}$ Olivier Huet, ${ }^{4}$ \\ Pierre François Perrigault, ${ }^{5}$ Claire Dahyot-fizelier, ${ }^{6}$ Philippe Seguin, ${ }^{7}$ \\ Tarek Sharshar, ${ }^{8}$ Thomas Geeraerts, ${ }^{9}$ Francis Remerand, ${ }^{10}$ Fanny Feuillet, ${ }^{11,12}$ \\ Karim Asehnoune, ${ }^{1}$ for the COBI group
}

To cite: Roquilly A, Lasocki S, Moyer JD, et al. COBI (COntinuous hyperosmolar therapy for traumatic Brain-Injured patients) trial protocol: a multicentre randomised open-label trial with blinded adjudication of primary outcome. BMJ Open 2017;7:e018035. doi:10.1136/ bmjopen-2017-018035

- Prepublication history and additional material for this paper are available online. To view please visit the journal (http:// dx.doi.org/10.1136/bmjopen2017-018035).

Congrès de la SFAR 2017, Paris

Received 5 June 2017

Revised 19 August 2017

Accepted 4 September 2017

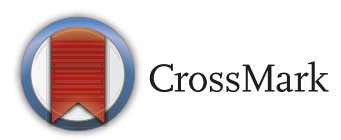

For numbered affiliations see end of article.

Correspondence to

Dr Antoine Roquilly;

antoine.roquilly@chu-nantes.Fr

\section{ABSTRACT}

Introduction Traumatic brain injury (TBI) is a major cause of death and severe prolonged disability. Intracranial hypertension (ICH) is a critical risk factor of bad outcomes after TBI. Continuous infusion of hyperosmolar therapy has been proposed for the prevention and the treatment of ICH. Whether an early administration of continuous hyperosmolar therapy improves long-term outcomes of patients with $T B I$ is uncertain. The aim of the COBI study (number clinicaltrial.gov 03143751, pre-results stage) is to assess the efficiency and the safety of continuous hyperosmolar therapy in patients with TBI.

Methods and analysis The $\mathrm{COBI}$ (COntinuous hyperosmolar therapy in traumatic Brain-Injured patients) trial is a multicentre, randomised, controlled, open-label, two-arms study with blinded adjudication of primary outcome. Three hundred and seventy patients hospitalised in intensive care unit with a TBI (Glasgow Coma Scale $\leq 12$ and abnormal brain CT scan) are randomised in the first 24 hours following trauma to standard care or continuous hyperosmolar therapy $(20 \% \mathrm{NaCl})$ plus standard care. Continuous hyperosmolar therapy is maintained for at least 48 hours in the treatment group and continued for as long as is necessary to prevent $\mathrm{ICH}$. The primary outcome is the score on the Extended Glasgow Outcome Scale at 6 months. The treatment effect is estimated with ordinal logistic regression adjusted for prespecified prognostic factors and expressed as a common OR.

Ethics and dissemination The COBI trial protocol has been approved by the ethics committee of Paris lle de France VIII and will be carried out according to the principles of the Declaration of Helsinki and the Good Clinical Practice guidelines. The results of this study will be disseminated through presentation at scientific conferences and publication in peer-reviewed journals. The $\mathrm{COBI}$ trial is the first randomised controlled trial powered to investigate whether continuous hyperosmolar therapy in patients with TBI improve long-term recovery.
Strengths and limitations of this study

- This study is a multicentre, randomised, controlled and open-label trial adequately powered to determine whether continuous hyperosmolar therapy enhances neurological recovery 6 months after a traumatic brain injury.

- Limitations due to the open-label design are limited by a blinded adjudication of primary outcome.

- Treatment's benefits include reduced risk of intracranial hypertension, reduced risk of mortality and enhanced quality of life.

- This large study has the potential of changing international recommendations on the management of patients with moderate to severe traumatic brain injury.

Trial registration number Trial registration number is NCT03143751.

\section{INTRODUCTION}

Severe traumatic brain injuries (TBIs) are responsible of more than 5 million deaths every year worldwide. ${ }^{1}$ TBI is an extremely severe condition given that nearly one patient with severe TBI out of three dies in hospital and another third has a bad neurological outcome. $^{2}$ Therapies are therefore urgently needed to limit brain damages and thus to limit sequels and the tremendous medical costs of TBI. ${ }^{3}$

Prevention of pneumonia, ${ }^{4}$ of ventilator-induced lung injury ${ }^{5}$ and of delayed extubation $^{6}$ is critical in brain-injured patients. However, intracranial hypertension (ICH) 
remains the leading cause of secondary ischaemic brain injury and is the most frequent cause of death following head trauma. ${ }^{7}$ The frequency and cumulative duration of ICH episodes are independent risk factors for bad neurological outcomes. ${ }^{8} 9$ Although no randomised study has demonstrated the usefulness of intracranial pressure monitoring, ${ }^{10}$ the statistical association between the occurrence of ICH and the long-term outcome of patients with TBI justifies the international recommendations in favour of intracranial pressure monitoring and emphasises the importance of prevention and treatment of post-traumatic ICH. ${ }^{11}$

Continuous hyperosmolar therapy with infusion of hypertonic saline solutions $(3 \%-20 \%)$ has been proposed in patients with severe brain injuries to ensure continuous control of intracranial pressure. ${ }^{12}$ Continuous hyperosmolar therapy was first used for the treatment of refractory ICH. ${ }^{13}$ More recently, early administration of continuous hyperosmolar therapy, that is to say, in the first line of the curative treatment or even for the prevention of ICH in patients with TBI, has been implemented in clinical practice. ${ }^{14-17}$ However, to date, few data have demonstrated the efficacy of this treatment, and no study has investigated its effects on long-term outcomes.

The objective of the COBI (COntinuous hyperosmolar therapy in traumatic Brain-Injured patients) study is to ascertain whether or not the administration of early continuous hyperosmolar therapy with standard care compared with standard care alone improves neurological outcome at 6 months after injury in patients with moderate or severe TBI. We are reporting the version 2 of the protocol (27 April 2017).

\section{METHODS AND DESIGN \\ Hypothesis}

Patients treated with early continuous hyperosmolar therapy have reduced morbidity and mortality rates compared with those receiving standard care alone after TBI.

\section{Research questions}

1. Does early continuous hyperosmolar therapy enhance long-term recovery after TBI assessed by the Extended Glasgow Outcome Scale (GOS-E) questionnaire?

2. Does early continuous hyperosmolar therapy reduce mortality rates and enhance the quality of life at 3 and 6 months after TBI assessed by the GOS-E questionnaire?

3. Does early continuous hyperosmolar therapy prevent ICH and reduce ICH severity?

\section{Design}

The COBI study is a multicentre, randomised, open-label, controlled trial with blinded adjudication of primary outcome.

\section{Ethics}

The institutional review board of Ile de France VIII (France) approved the study protocol (May 2017). A patient's legal surrogate provides written consent for participation when possible. Patients are eligible to be enrolled without the provision of legal surrogate consent if next of kin cannot be informed in the maximal delay for the inclusion. Patients who recover sufficient capacity to provide consent are asked to consent to continue in the trial. COBI is conducted in accordance with the Declaration of Helsinki and is registered on May 2017 at http://clinicaltrial.gov/ with trial registration NCT03143751.

\section{Study population}

Investigators screen patients with consecutive moderate to severe TBI defined as the association of a Coma Glasgow Scale $\leq 12$ (considering the worst score observed in the first 24 hours following trauma) together with a traumatic abnormal brain CT scan (extradural haematoma, subdural haematoma, subarachnoid haemorrhage, brain contusion, brain haematoma, brain oedema or skull fracture). Inclusions criteria are: moderate (Glasgow Coma Scale 9-12) to severe (Glasgow Coma Scale 3-8) TBI, in the first 24 hours following trauma, age between 18 and 80 years and informed consent from a next of kin. Exclusions criteria are: dependence for daily activity, Coma Glasgow Scale of 3 and fixed dilated pupils, associated cervical spine injury, imminent death and do-not-resuscitate orders and pregnancy.

\section{Setting}

The study involved 10 French neuro-intensive care units from 10 university hospitals, each centre caring for more than 50 patients with moderate to severe TBI every year.

\section{Treatment allocation}

Patients eligible for inclusion are randomised within the first 24 hours after trauma. Patients are randomised in a 1:1 ratio and stratified according to the Glasgow Coma Scale (3-8 or 9-12) and administration of boluses of hyperosmolar therapy before inclusion. Randomisation is made by a computerised number generator list provided by a statistician not involved in the determination of eligibility or in the assessment of outcomes. All assignments are made through a dedicated, password-protected, SSL-encrypted website. Patients are randomised in the first 24 hours following trauma to standard care (control group) or continuous hyperosmolar therapy plus standard care (intervention group).

\section{Masking protocol}

It is not possible to blind local investigators to allocation as it is obvious clinically which patients are receiving continuous hyperosmolar therapy. Blinding of outcome data assessment is, however, ensured as the GOS-E questionnaire is evaluated over the phone by a research assistant not aware of patient assignment. ${ }^{18} 19$ 


\section{Continuous hyperosmolar therapy for traumatic brain}

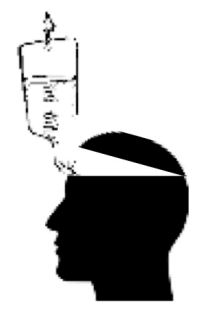

Continuous hyperosmolar therapy

\section{Initiation of the treatment: 1-hour bolus}

\begin{tabular}{|c|c|}
\hline $\begin{array}{l}\text { Blood level of sodium before } \\
\text { inclusion }\end{array}$ & Amount of sodium chloride \\
\hline $150 \mathrm{mmol} / \mathrm{L}<\mathrm{Na}+$ & No bolus \\
\hline $145<\mathrm{Na}+\leq 150 \mathrm{mmol} / \mathrm{L}$ & $7.5 \mathrm{~g}(37.5 \mathrm{~mL}$ of $\mathrm{NaCl} 20 \%)$ over 1 hour \\
\hline $\mathrm{Na}+\leq 145 \mathrm{mmol} / \mathrm{L}$ & $15 \mathrm{~g} \mathrm{(75} \mathrm{mL} \mathrm{of} \mathrm{NaCl} 20 \%)$ over 1 hour \\
\hline
\end{tabular}

\section{Continuous infusion of $\mathrm{NaCl} 20 \%$ initiated immediately after the 1-hour bolus, for a minimal duration of $\mathbf{4 8}$ hours}
a. Continuous infusion of $\mathrm{NaCl}: 1 \mathrm{~g} /$ hour $(5 \mathrm{~mL} /$ hour of $\mathrm{NaCl} 20 \%$ )
b. Biological monitoring
i. Blood level of sodium (x3/day)
ii. Blood osmolality (x1/day)

c. Dose-adaptation

\begin{tabular}{|c|c|}
\hline Blood level of sodium inclusion & Amount of sodium chloride \\
\hline $155 \mathrm{mmol} / \mathrm{L}<\mathrm{Na}+$ & Stop \\
\hline $150<\mathrm{Na}+\leq 155 \mathrm{mmol} / \mathrm{L}$ & $0.5 \mathrm{~g} /$ hour $(2.5 \mathrm{~mL} /$ hour of $\mathrm{NaCl} 20 \%)$ \\
\hline $\mathrm{Na}+\leq 150 \mathrm{mmol} / \mathrm{L}$ & $1 \mathrm{~g} /$ hour $(5 \mathrm{~mL} /$ hour of $\mathrm{NaCl} 20 \%)$ \\
\hline
\end{tabular}

\section{Treatment discontinuation}

a. Biological monitoring: Blood level of sodium x 2-3/day for 48 hours.

\begin{tabular}{|l|l|}
\hline \multicolumn{1}{|c|}{ Blood level of sodium inclusion } & \multicolumn{1}{|c|}{ Amount of sodium chloride } \\
\hline $140 \mathrm{mmol} / \mathrm{L}<\mathrm{Na}+$ & Stop \\
\hline $\mathrm{Na}+\leq 140 \mathrm{mmol} / \mathrm{L}$ & $5 \mathrm{~g}(25 \mathrm{~mL}$ of $\mathrm{NaCl} 20 \%)$ over 1 hour \\
\hline
\end{tabular}

Figure 1 Protocol for the dose adaptation of the continuous hyperosmolar therapy.

\section{Procedures}

Within 24hours of the estimated time of TBI, a 1-hour bolus infusion (7.5-15g of $20 \% \mathrm{NaCl}$ depending on the blood level of sodium before inclusion) is realised immediately after the recruitment into the study. Thereafter, continuous hyperosmolar therapy is pursued with a continuous intravenous infusion $(0.5-1 \mathrm{~g} /$ hour of $20 \% \mathrm{NaCl}$ ) adapted to the blood level of sodium measured every 8 hours (figures 1 and 2). Continuous hyperosmolar therapy is pursued for at least 48 hours in the intervention group and continued for as long as necessary to control ICH (if monitored). If the $20 \% \mathrm{NaCl}$ infusion is continued after 48 hours for ongoing ICH, it can be stopped be when all the stage four therapies are suspended for 12 hours or more. $20 \% \mathrm{NaCl}$ is infused on a central venous catheter. Blood level of sodium is monitored every 8 hours during the treatment and for 48 hours after the complete cessation.

Site medical teams agree to adhere to the revised Brain Trauma Foundation guidelines ${ }^{11}$; however, they retain full independent control of management of every patient's TBI. Both arms received standard treatments (see online supplementary methods: standard of care for TBI). In case of ICH, the groups receive standard treatments, 


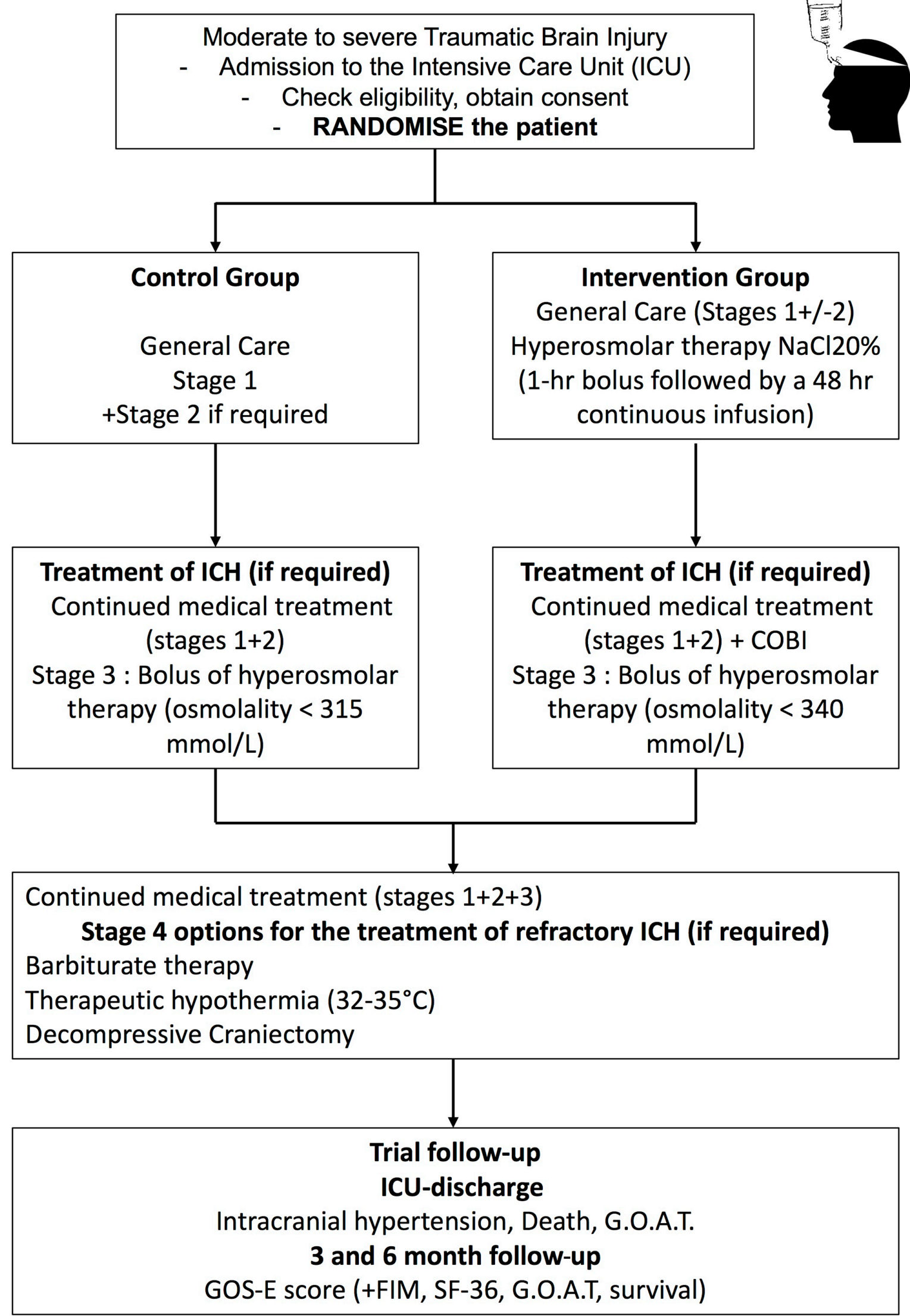

Figure 2 Stages of therapeutic management and trial follow-up. FIM, functional independence measure; GOAT, Galveston Orientation and Amnesia Test; GOS-E, Extended Glasgow Outcome Scale; ICH, intracranial hypertension; SF-36, Short Form 36. 
including bolus of hyperosmolar therapy (mannitol $0.25-1 \mathrm{~g} / \mathrm{kg}$ body weight or $100 \mathrm{~mL}$ of $7.45 \%$ hypertonic saline) in case of ICH, but without continuous infusion of hyperosmolar therapy for the control group (figure 2).

\section{Protocol drop-out}

Clinicians can use continuous infusion of hyperosmolar therapy in the control group only in case of ICH refractory to other therapies (rescue treatment). Patients of the control group treated by continuous hyperosmolar therapy in the case of refractory ICH will be kept in analysis and remain with the control group.

\section{Study endpoints}

The primary outcome is the GOS-E questionnaire at sixth month.

Secondary outcomes are 3-month and 6-month mortality rates, 3-month GOS-E questionnaire, rate of patients with post-traumatic amnesia (Galveston Orientation and Amnesia Test (GOAT)), 3-month and 6-month quality-of-life assessments (functional independence measure (FIM) and Short Form 36 (SF-36)), blood level of natremia, blood osmolarity, intracranial pressure control (time course of intracranial pressure over the first 28 days), rate and severity of ICH (mean durations of stage 3 or 4 treatments and daily percentages of patients receiving stage 3 or 4 treatments; see figure 2) and tolerance (acute kidney injury, thromboembolic events).

\section{Follow-up data}

A letter is sent by post to the patient's general practitioner to inform them of the patient's involvement in the COBI trial. Patient outcome is assessed 6 months after injury using the GOS-E, the FIM and the SF-36 questionnaires. It is critical to obtain this information since GOS-E is the primary outcome. If the patient is discharged alive from hospital, a member of the trial team will telephone the patient's family doctor to find out their vital status before any questionnaires are sent to the patient. Then, one trained research assistant from the coordinating centre who is unaware of the study group assignments will ask enrolled patients or, if appropriate, relatives or caregivers to answer a structured questionnaire by telephone. ${ }^{1920}$

\section{Data collection and checking}

An online case-report form is used for collection of data. Blinded and patient identifiable data are stored separately in secure databases. All patient identifiable data are stored by the coordinating centre. The following variables are collected: demographics, Glasgow Coma Scale (worst score before inclusion), neuro-surgery, intracranial pressure (every 8 hours for 5 days, then daily at 08:00), mean arterial pressure (every 8 hours for 5 days, then daily at 08:00), natremia (every 8 hours for 5 days, then daily at 08:00), blood osmolality (daily for 5 days), treatment of ICH (daily administration of stage 3-4 therapies, if any), decision of care withdrawal, ICU hospitalisation and survival. At 3 and 6 months, GOS-E, FIM and SF-36 and survival are recorded. Staff in Nantes works closely with local investigators to obtain data that are as complete and accurate as possible.

\section{Study monitoring}

The study will be monitored on behalf of the promoter (Nantes University Hospital). Site staff will be available to facilitate the monitoring visits and ensure that all required documentation is available for review. Study initiation visits are carried out at all sites before recruitment commences at that site. During regular monitoring visits realised throughout the duration of the trial, an independent research assistant will carry out Source Data Verification of trial data, verify informed consent forms and ensure the completeness of the Investigator Site Files.

\section{Study oversight}

Study sponsor is the Nantes University Hospital (5 allee de l'île Gloriette, 44000 Nantes, drc-nantes@chu-nantes. fr). Experienced research staff monitored the study for quality, the integrity of data in all the participating centres. Serious adverse events and unexpected related or possibly related serious events are reported blinded to the promotor within respectively 24 hours or 7 days. An independent data and safety monitoring board (DSMB) is appointed by the sponsor. The DSMB is made up of three people with no connection to the research, including one clinician specialising in the management of TBI, one pharmacovigilance specialist and a methodologist/biostatistician, particularly in the case of interim analysis. Before the first inclusion and every 100 inclusions, the DSMB looks over the ethics in accordance with the Declaration of Helsinki, monitors patient safety and reviews safety issues as the study progresses. The DSMB makes recommendations to the sponsor about the continuation, modification or termination of the research. The recommendations that the DSMB can make are:

- to continue the research with no modifications

- to continue the research with a modification to the protocol and/or to the monitoring of subjects

- to temporarily halt inclusions

- to permanently terminate the research in light of serious adverse reactions.

The DSMB has a consultative role in advising the sponsor on safety issues such as tolerance and reassessment of the benefit to risk ratio during the research. Trial recruitment can be stopped by the promotor on the advice of the DSMB in case of safety concern.

\section{Roles of the sponsor and of the funder}

The sponsor and of the funder had no role in the design or conduct of the study, the data analysis, the writing of the manuscript or in the decision to submit the manuscript.

\section{Statistical consideration}

GOS-E questionnaire at 6 months is the primary outcome. In pilot randomised clinical trials, continuous osmotherapy has reduced the rates of death by $20 \%$ and of ICH by $30 \% .{ }^{17}$ A relative decrease in the risk of poor neurological recovery of $20 \%$ seems reasonable. The 
proportion of patients with GOS-E less than or equal to 5 (poor neurological recovery) in the control group is expected to reach $70 \%{ }^{21}$

To demonstrate a relative decrease in poor neurological recovery of $20 \%(56 \%$ GOS-E $\leq 5)$ in the intervention group, versus $70 \%$ in the control group, 370 patients should be included to ensure $80 \%$ with an alpha risk of 0.05 (185 per randomisation arm). The ordinal approach for the analysis of the primary endpoint will increase the statistical power of the study. However, it is not advisable to reduce the number of patients to be included. ${ }^{22}$

\section{Preplanned primary analysis}

The analysis will be undertaken on the intention-totreat' principle, including all randomly assigned patients except those who withdrew consent for use of all trial data and those who did not meet the inclusion criteria.

For the primary analysis, the distribution of the 6 -month GOS-E scores between the two groups (intervention vs control) will be compared with the use of ordinal logistic regression adjusted for key baseline covariates (Age, Glasgow Coma Scale, pupillary reactivity, hypotension, hypoxia, anaemia and brain CT classification) $)^{2324}$ and with covariates used for stratification of the randomisation (bolus of hyperosmolar therapy before inclusion).

Planned subgroup analyses are:

- strates of randomisation: severe TBI (Glasgow Coma Scale 3-8) and moderate TBI (Glasgow Coma Scale 9-12)

- bolus of hyperosmolar therapy prior to inclusion

- neurosurgical intervention prior to inclusion

- blood level of sodium before inclusion $(<138 \mathrm{mmol} / \mathrm{L}$, $138-145 \mathrm{mmol} / \mathrm{L}$ and $>145 \mathrm{mmol} / \mathrm{L}$ )

- pupil reactivity

- age (<40 years, $40-60$ years and $>60$ years)

- time to inclusion (<8 hours, 8-16hours and 16-24 hours between trauma and inclusion).

The proportion of patients with good outcome (GOS-E 6-8) will also be assessed using an uncorrected and adjusted (using the same adjustment as the primary analysis) logistic regression and the results will be reported as an unadjusted and adjusted risk ratio with associated 95\% CIs.

Continuous variables are reported as mean (SD) or median (IQR). Proportions are reported as raw numbers (percentages). We will assess secondary outcomes using uncorrected regression models (linear or logistic) and report the results as an unadjusted risk ratio with associated $95 \%$ CIs. Time course of the blood levels of sodium and of plasma osmolarity as well as intracranial pressure values will be analysed by linear mixed models taking into account the effects time and treatment.

No interim efficacy analysis will be performed so that no adjustment is required to the final $\mathrm{p}$ value to allow for the multiple testing. The DSMB will only analyse safety data.

\section{Data sharing}

The principal investigator will have access to the final trial data set. Patient level data and/or full dataset and/or statistical code will be available on request to the corresponding author. Consent was not obtained, but the presented data are anonymised and risk of identification is low and the potential benefits of sharing these data outweigh the potential harms.

\section{DISCUSSION}

The COBI trial is the first randomised controlled study powered to investigate early continuous hyperosmolar therapy in patients with TBI.

We will include patients with moderate to severe TBI (Glasgow Coma Scale 3-12). The International IMPACT Working Group has made recommendations on the methodology of clinical trials aimed at improving the power of neuro-reanimation trials. ${ }^{25}$ It is thus recommended to use inclusion criteria as broad as possible, as long as they are compatible with the mechanisms of action of the evaluated intervention. This strategy maximises recruitment rates and improves the generalisation of results. Following this recommendation and since the secondary occurrence of ICH cannot be excluded in patients with moderate head trauma (Glasgow score 9-12), ${ }^{26}$ patients with moderate to severe head trauma will be included in the COBI trial. The inclusion of patients with moderate TBI can theoretically decrease the study power. However, in line with the demonstration that $44 \%$ of patients with moderate TBI have incomplete recovery at 6 months, ${ }^{27}$ Nichol et al reported in a recent randomised international trial that $70 \%$ of patients with moderate to severe TBI have poor outcome at 6 months (GOS-E 1-5). ${ }^{21}$ This high incidence rate of the primary endpoint in a population with moderate to severe TBI will result in a study of high clinical relevance and statistical power.

We defined the GOS-E at 6 months as the primary criteria. The use of mortality as a primary endpoint is not recommended in neuro-resuscitation studies because a strategy that increases survival at the cost of heavy sequelae (eg, pauci-relational states) would not be recommended. For example, erythropoietin (EPO) therapy has recently been shown to improve the survival of TBI without increasing the percentage of patients with good neurological recovery. ${ }^{21}$ Therefore, EPO is not recommended at this time. ${ }^{11} 28$ The IMPACT group recommended choosing a primary endpoint measuring the autonomy or quality of life of patients. Among the scores measuring functional recovery and quality of life after head trauma, GOS-E is the most reliable, validated and most used score in randomised trials in TBI. ${ }^{19}$ Finally, mortality is not a competitive with the primary outcome since the outcome 'death' is included in the GOS-E (death=1).

The GOS-E can be evaluated between 3 and 12 months after the trauma. Between 6 and 12 months, the autonomy scores have changed little, ${ }^{2}$ and the follow-up of patients will therefore be limited to 6 months in this 
study (primary endpoint), with an analysis also at 3 months (secondary endpoint). Previous findings indicate that structured interview over the telephone provides reliable assessment of the GOS-E as compared with in-person contact. ${ }^{29}{ }^{30}$ Due to feasibility concerns, we thus have decided that all the included patients will be followed up over the telephone by one trained research assistant of the coordinating centre.

In accordance with the IMPACT recommendations, for the primary statistical analysis of the GOS-E, we will use an ordinal logistic regression model, with adjustment to prespecified key cofactors. ${ }^{25}$ This strategy will increase the statistical power of our study ${ }^{22}$ and has been used in several recent studies in neuro-resuscitation. ${ }^{31-33}$ It also has the advantage of not having to use non-consensual definitions of a 'good' and 'bad' neurological recovery necessary for the conventional dichotomous analysis of GOS-E. Finally, the adjustment to predefined covariates ${ }^{23}$ will mitigate the effects of heterogeneity resulting from the use of broad inclusion criteria (Glasgow Coma Scale $3-13) .^{34}$

\section{Trial status}

The trial has already achieved many milestones. Sponsorship has been agreed: the trial is sponsored by the French Ministry of Health. Insurance for non-negligent harm has been provided by University Hospital of Nantes (France). Research ethics committee approval was obtained in June 2017. It is registered with the American registry of trials (https://clinicaltrials. gov/; NCT03143751). The current emphasis is on developing the recruitment infrastructure, which is ongoing, and developing the follow-up infrastructure. No patient has yet been included, and expected starting point of the study is September 2017.

The principal investigator (AR), the scientific expert (KA) and the statistician (FF) will write the first draft of the manuscript. All the co-authors (investigators who had realised not less than 20 inclusions) will append and approve the final manuscript before the submission. No professional writer will be used.

\section{Ethics and dissemination}

The COBI trial protocol has been approved by the ethics committee of Paris Ile de France VIII and will be carried out according to the principles of the Declaration of Helsinki and the Good Clinical Practice guidelines. The results of this study will be disseminated through presentation at scientific conferences and publication in peer-reviewed journals. The COBI trial is an investigator-initiated randomised controlled trial powered to test the hypothesis that the continuous hyperosmolar therapy in patients with moderate to severe TBI enhances neurological outcomes. The results of the COBI Trial will be relevant to clinicians who look after patients with moderate to severe TBI. An ancillary study will assess the long-term neurological and psychological recovery of the next of kin of included patients.

\section{Author affiliations}

${ }^{1}$ Anaesthesia Intensive Care Unit, Centre Hospitalier Universitaire, Nantes, France ${ }^{2}$ Anaesthesia Intensive Care Unit, Centre Hospitalier Universitaire, Angers, France ${ }^{3}$ Anaesthesia Intensive Care Unit, Centre Hospitalier Universitaire de Beaujon (AP/ $\mathrm{HP})$, Beaujon, France

${ }^{4}$ Intensive Care Unit, Anaesthesia, Centre Hospitalier Universitaire, Brest, France ${ }^{5}$ Anaesthesia Intensive Care Unit, Centre Hospitalier Universitaire, Montpellier, France

${ }^{6}$ Anaesthesia and Intensive Care Unit, Centre Hospitalier Universitaire, Poitiers, France

${ }^{7}$ Anaesthesia and Intensive Care Unit, Centre Hospitalier Universitaire, Rennes, France

${ }^{8}$ Anaesthesia and Intensive Care Unit, Hôpital Saint Anne (AP/HP), Paris, France ${ }^{9}$ Department of Anesthesiology and Critical Care, University Hospital of Toulouse, TONIC (Toulouse Neurolmaging Center), University Toulouse 3-Paul Sabatier, Inserm, France

${ }^{10}$ Service anesthésie Réanimation 2, Centre Hospitalier Régional Universitaire de Tours, Université $\mathrm{F}$ Rabelais, Tours, France

${ }^{11}$ Département Promotion, Centre Hospitalier Universitaire de Nantes, Plateforme de Méthodologie et de Biostatistique, Direction de la Recherche, Nantes, France

${ }^{12}$ Université de Nantes, Université de Tours, INSERM, SPHERE U1246, Nantes, France

Acknowledgements We thank patients and relatives, physicians, nursing staff and clinical research associates of the participating centres for their involvement in this study.

Collaborators Alphabetical order of centres of the COBI group: Intensive Care Unit of Angers (France): Thomas Gaillard (MD) (Thomas.gaillard@chu-angers. fr); Intensive Care Unit of Beaujon (AP, HP, France): Catherine Paugam-Burtz (MD, $\mathrm{PhD}$ ) (catherine.paugam@bjn.aphp.fr); Intensive Care Unit of Brest (France): Véronique Vermeersch (MD) (veronique.vermeersch@chu-brest.fr); Intensive Care Unit of Nantes (France): Raphael Cinotti (MD) (raphael.cinotti@chu-nantes.fr), Dominique Demeure Dit Latte (MD) (dominique.demeureditlatte@chu-nantes.fr), Pierre Joachim Mahe (MD) (pierrejoachim.mahe@chu-nantes.fr); Intensive Care Unit of Poitiers (France): Oliver Mimoz (MD) (0.mimoz@chu-poitiers.fr); Intensive Care Unit of Rennes (France): Sonia Isslame (MD) (sonia.isslame@chu-rennes.fr), Yoann Launey (MD) (yoann.launey@chu-rennes.fr), Nicolas Nesseler (MD) (Nicolas. nesseler@chu-rennes.fr); Intensive Care Unit of Toulouse (France): Segolene Mrozek (MD) (segolene.mrozek@chu-toulouse.fr), Fouad Marhar (MD) (fouad.marhar@ chu-toulouse.fr); Intensive Care Unit of Tours (France): Djillali Elaroussi (MD) (djillali. elaroussi@chu-tours.fr).

Contributors AR conceived the study, coordinated its design and drafted the manuscript. AR and KA wrote the manuscript. SL, JDM, OH, PFP, CD-F, PS, TS, TG, FR and FF read and were involved in critical appraisal and revision of the manuscript. FF provided statistical expertise. All authors approved the final manuscript prior to submission.

Funding This study is an investigator-initiated trial, funded by a grant from the French Ministry of Health (PHRCI 2016, RC16_0474).

Competing interests None declared.

Patient consent Obtained.

Ethics approval Institutional Review Board of Ile de France VIII.

Provenance and peer review Not commissioned; externally peer reviewed.

Data sharing statement The principal investigator will have access to the finaltrial data set. Patient level data and/or full datasetand/or statistical code will be available on request tothe corresponding author. Consent was not obtained,but the presented data are anonymised and risk ofidentification is low and the potential benefits ofsharing these data outweigh the potential harms.

Open Access This is an Open Access article distributed in accordance with the Creative Commons Attribution Non Commercial (CC BY-NC 4.0) license, which permits others to distribute, remix, adapt, build upon this work non-commercially, and license their derivative works on different terms, provided the original work is properly cited and the use is non-commercial. See: http://creativecommons.org/ licenses/by-nc/4.0/

(C) Article author(s) (or their employer(s) unless otherwise stated in the text of the article) 2017. All rights reserved. No commercial use is permitted unless otherwise expressly granted. 


\section{REFERENCES}

1. Mokdad AH, Forouzanfar MH, Daoud F, et al. Global burden of diseases, injuries, and risk factors for young people's health during 1990-2013: a systematic analysis for the Global Burden of Disease Study 2013. Lancet 2016;387:2383-401.

2. Sandhaug M, Andelic N, Langhammer B, et al. Functional level during the first 2 years after moderate and severe traumatic brain injury. Brain Inj 2015;29:1431-8.

3. Olesen J, Gustavsson A, Svensson M, et al. The economic cost of brain disorders in Europe. Eur J Neurol 2012;19:155-62.

4. Asehnoune K, Seguin P, Allary J, et al. Hydrocortisone and fludrocortisone for prevention of hospital-acquired pneumonia in patients with severe traumatic brain injury (Corti-TC): a double-blind, multicentre phase 3, randomised placebo-controlled trial. Lancet Respir Med 2014;2:706-16.

5. Asehnoune K, Mrozek S, Perrigault PF, et al. A multi-faceted strategy to reduce ventilation-associated mortality in brain-injured patients. The BI-VILI project: a nationwide quality improvement project. Intensive Care Med 2017;43:957-70.

6. Roquilly A, Cinotti R, Jaber S, et al. Implementation of an evidencebased extubation readiness bundle in 499 brain-injured patients. a before-after evaluation of a quality improvement project. Am J Respir Crit Care Med 2013;188:958-66.

7. Rosenfeld JV, Maas Al, Bragge P, et al. Early management of severe traumatic brain injury. Lancet 2012;380:1088-98.

8. Struchen MA, Hannay HJ, Contant CF, et al. The relation between acute physiological variables and outcome on the Glasgow Outcome Scale and Disability Rating Scale following severe traumatic brain injury. J Neurotrauma 2001;18:115-25.

9. Badri S, Chen J, Barber J, et al. Mortality and long-term functional outcome associated with intracranial pressure after traumatic brain injury. Intensive Care Med 2012;38:1800-9.

10. Chesnut RM, Temkin N, Carney N, et al. A trial of intracranial-pressure monitoring in traumatic brain injury. N Engl J Med 2012;367:2471-81.

11. Carney N, Totten AM, O'Reilly C, et al. Guidelines for the Management of Severe Traumatic Brain Injury, Fourth Edition. Neurosurgery 2016:1-10.

12. Tyagi R, Donaldson $\mathrm{K}$, Loftus $\mathrm{CM}$, et al. Hypertonic saline: a clinical review. Neurosurg Rev 2007;30:277289-9090.

13. Roquilly A, Mahe PJ, Latte DD, et al. Continuous controlled-infusion of hypertonic saline solution in traumatic brain-injured patients: a 9-year retrospective study. Crit Care 2011;15:R260.

14. Froelich $\mathrm{M}, \mathrm{Ni} \mathrm{Q}$, Wess $\mathrm{C}$, et al. Continuous hypertonic saline therapy and the occurrence of complications in neurocritically ill patients. Crit Care Med 2009;37:1433-41.

15. Hauer EM, Stark D, Staykov D, et al. Early continuous hypertonic saline infusion in patients with severe cerebrovascular disease. Crit Care Med 2011;39:1766-72.

16. Tan SK, Kolmodin L, Sekhon MS, et al. The effect of continuous hypertonic saline infusion and hypernatremia on mortality in patients with severe traumatic brain injury: a retrospective cohort study. Can $J$ Anaesth 2016;63:664-73.

17. Ichai C, Payen JF, Orban JC, et al. Half-molar sodium lactate infusion to prevent intracranial hypertensive episodes in severe traumatic brain injured patients: a randomized controlled trial. Intensive Care Med 2013;39:1413-22.
18. Assessing disability after head injury: improved use of the Glasgow Outcome Scale. J Neurosurg 2009;89:939-43.

19. Nichol AD, Higgins AM, Gabbe BJ, et al. Measuring functional and quality of life outcomes following major head injury: common scales and checklists. Injury 2011;42:281-7.

20. Fayol $P$, Carrière $H$, Habonimana $D$, et al. Version française de I"entretien structuré pour l"échelle de devenir de Glasgow (GOS): recommandations et premières études de validation. Annales de Réadaptation et de Médecine Physique 2004;47:142-56.

21. Nichol A, French C, Little L, et al. Erythropoietin in traumatic brain injury (EPO-TBI): a double-blind randomised controlled trial. Lancet 2015;386:2499-506.

22. Roozenbeek B, Lingsma HF, Perel $P$, et al. The added value of ordinal analysis in clinical trials: an example in traumatic brain injury. Crit Care 2011;15:R127.

23. Steyerberg EW, Mushkudiani N, Perel P, et al. Predicting outcome after traumatic brain injury: development and international validation of prognostic scores based on admission characteristics. PLoS Med 2008;5:e165.

24. Maas Al, Menon DK, Lingsma HF, et al. Re-orientation of clinical research in traumatic brain injury: report of an international workshop on comparative effectiveness research. J Neurotrauma 2012;29:32-46.

25. Maas Al, Steyerberg EW, Marmarou A, et al. IMPACT recommendations for improving the design and analysis of clinical trials in moderate to severe traumatic brain injury. Neurotherapeutics 2010;7:127-34.

26. Bouzat P, Almeras L, Manhes $P$, et al. Transcranial doppler to predict neurologic outcome after mild to moderate traumatic brain injury. Anesthesiology 2016;125:346-54.

27. van der Naalt J, Timmerman ME, de Koning ME, et al. Early predictors of outcome after mild traumatic brain injury (UPFRONT): an observational cohort study. Lancet Neurol 2017;16:532-40.

28. Geeraets T, Velly L, Abdennour L, et al. Management of severe traumatic brain injury (first 24 hours). http://sfar.org/prise-en-chargedes-traumatises-craniens-graves-a-la-phase-precoce/ (accessed 15 Jul 2017).

29. Pettigrew LE, Wilson JT, Teasdale GM. Reliability of ratings on the Glasgow Outcome Scales from in-person and telephone structured interviews. J Head Trauma Rehabil 2003;18:252-8.

30. Wilson JTL, Pettigrew LEL, Teasdale GM. Structured interviews for the Glasgow Outcome Scale and the Extended Glasgow Outcome Scale: guidelines for their use. J Neurotrauma 1998;15:573-85.

31. Anderson CS, Heeley E, Huang Y, et al. Rapid blood-pressure lowering in patients with acute intracerebral hemorrhage. $N$ Engl J Med 2013;368:2355-65.

32. Cooper DJ, Rosenfeld JV, Murray L, et al. Decompressive craniectomy in diffuse traumatic brain injury. $N$ Engl J Med 2011;364:1493-502.

33. Andrews PJ, Sinclair HL, Rodriguez A, et al. Hypothermia for intracranial hypertension after traumatic brain injury. $N$ Engl J Med 2015;373:2403-12.

34. Maas Al, Roozenbeek B, Manley GT. Clinical trials in traumatic brain injury: past experience and current developments. Neurotherapeutics 2010;7:115-26. 